\title{
FREQUÊNCIA DA INCONTINÊNCIA URINÁRIA EM MULHERES NA IDADE PRODUTIVA
}

\section{FREQUENCY OF URINARY INCONTINENCE IN WOMEN IN THE PRODUCTIVE AGE}

\section{FRECUENCIA DE LA INCONTINENCIA URINARIA EN MUJERES EN LA EDAD PRODUCTIVA}

\author{
Mara Angélica Dantas ${ }^{1}$, Cássio Dias², Ellany Gurgel Cosme do Nascimento ${ }^{3}$
}

Como citar esse artigo: Dantas MA, Dias C, Nascimento EGC. Frequência da incontinência urinária em mulheres na idade produtiva. Rev Enferm Atenção Saúde, v. 9, n. 2, 16-27, 2020. DOI: 10.18554/reas.v9i2.3521

\begin{abstract}
RESUMO
Introdução: A Incontinência Urinária é definida como a queixa de qualquer perda involuntária de urina. Método: Trata-se de uma pesquisa descritiva exploratória, transversal e com abordagem quantitativa. $\mathrm{O}$ estudo foi realizado na cidade de Pilões/RN. Participaram 300 mulheres. A coleta de dados foi mediante questionário, que foi auto aplicado com exceção dos casos de mulheres analfabetas. Resultados: A frequência de IU feminina encontrada foi de $8 \%$. A média de idade foi de 38,8 anos. A média de vezes que as mulheres com IU urinam durante o dia foi de 6,5 $\pm 1,6$ vezes e durante a noite foi de 1,8 vezes. Conclusões: $O$ presente estudo nos proporcionou conhecer a frequência da incontinência urinária em mulheres em idade produtiva na cidade de Pilões/RN. O mesmo nos levou a concluir que infelizmente todas as mulheres que não tem conhecimento sobre o que é a IU.
\end{abstract}

Descritores: Incontinência Urinária; Enfermagem; Saúde da mulher.

\begin{abstract}
Introduction: Urinary Incontinence is defined as the complaint of any involuntary loss of urine. Method: This is an exploratory, cross-sectional descriptive study with a quantitative approach. The study was carried out in the city of Pilões / RN. 300 women participated. Data collection was carried out through a questionnaire, which was self-administered with the exception of cases of illiterate women. Results: The frequency of female UI found was $8 \%$. The average age was 38.8 years. The average number of times women with UI urinate during the day was $6.5 \pm$ 1.6 times and during the night it was 1.8 times. Conclusions: The present study allowed us to know the frequency of urinary incontinence in women of working age in the city of Pilões / $\mathrm{RN}$. The same led us to conclude that unfortunately all women who are unaware of what UI is. Descriptors: Urinary incontinence; Nursing; Women's health.
\end{abstract}

\section{RESUMEN}

Introducción: la incontinencia urinaria se define como la queja de cualquier pérdida involuntaria de orina. Método: Este es un estudio exploratorio, transversal, descriptivo con un enfoque cuantitativo. El estudio se realizó en la ciudad de Pilões / RN. Participaron 300 mujeres.

\footnotetext{
$\begin{array}{lllllll}1 & \text { Universidade } & \text { do } & \text { Rstado } & \text { do Nonde }\end{array}$ http://orcid.org/0000-0002-0344-5975

${ }^{2}$ Universidade Federal do Rio Grande do Norte. http://orcid.org/0000-0002-7811-0117

${ }^{3}$ Enfermeira. Doutora em Ciências da Saúde pela Universidade do Estado do Rio Grande do Norte. Professora Adjunta do Curso de Medicina da UERN. http://orcid.org/0000-0003-4014-6242
} 
La recolección de datos se realizó mediante un cuestionario, que se autoadministra con la excepción de los casos de mujeres analfabetas. Resultados: La frecuencia de IU femenina encontrada fue del $8 \%$. La edad promedio fue de 38.8 años. El número promedio de veces que las mujeres con IU orinan durante el día fue de $6.5 \pm 1.6$ veces y durante la noche fue de 1.8 veces. Conclusiones: El presente estudio nos permitió conocer la frecuencia de la incontinencia urinaria en mujeres en edad laboral en la ciudad de Pilões / RN. Lo mismo nos llevó a concluir que desafortunadamente todas las mujeres que desconocen qué es la IU.

Descriptores: Incontinencia urinaria; Enfermería; Salud de la mujer.

\section{INTRODUÇÃO}

A Incontinência Urinária (IU) é definida atualmente pela International Continence Society (ICS) como a queixa de qualquer perda involuntária de urina, valorizando $\mathrm{o}$ relato do usuário. $\mathrm{A}$ caracterização da IU ocorre de acordo com os eventos que levam à perda de urina, sendo classificada como IU de esforço perda urinária simultânea a esforço, exercício físico, tosse ou espirro; IU de urgência perda involuntária de urina acompanhada ou imediatamente precedida por súbito e incontrolável desejo de urinar, difícil de ser adiado ou mista quando há sinais e sintomas dos dois tipos relatados acima. $^{1}$

A IU atinge no mundo mais de 50 milhões de pessoas de todas as idades, principalmente as mulheres, que experimentam a patologia com uma frequência duas vezes maior que os homens. ${ }^{2,3,4}$ Segundo a Sociedade Brasileira de Urologia, cerca de $40 \%$ das mulheres se queixam de algum tipo de incontinência urinária após a menopausa. Entre aquelas que estão no período reprodutivo, estima-se que $25 \%$ apresentam a disfunção. ${ }^{5}$

Vale salientar que a experi40ência com episódios de perda urinária é uma condição que ocorre não somente entre mulheres idosas, mas também entre mulheres jovens e na meia-idade. Estudo epidemiológico revelou uma prevalência da IU de 26,5\% em mulheres de 35 a 64 anos e $41 \%$ naquelas acima de 65 anos. Outros trabalhos descrevem uma prevalência de 49,6\% em mulheres acima dos 20 anos e $45 \%$ nas que se encontram entre 30 e 90 anos. $^{6}$

Os fatores de risco citados para o desenvolvimento da incontinência urinária de esforço incluem idade avançada, etnia branca, obesidade, partos vaginais, deficiência estrogênica, menopausa, condições associadas a aumento da pressão intra-abdominal, tabagismo, doenças do colágeno, neuropatias e histerectomia prévia. $^{7}$

Além do desconforto higiênico, a IU exerce múltiplos efeitos sobre as atividades diárias, leva a mulher a restringir ou 
diminuir as suas atividades sociais e físicas, progredindo para alterações a nível emocional, incluindo a baixa autoestima, depressão, vergonha, medo, isolamento e a autopercepção do estado de saúde. Seu efeito psicossocial pode ser mais devastador do que as consequências sobre a saúde. ${ }^{8,2} \mathrm{~A}$ depressão e a doença do pânico são altamente prevalentes em mulheres com IU. $^{6}$

Mediante esta problemática, decidiuse conhecer a frequência da incontinência urinária em mulheres em idade produtiva e o conhecimentos das mesmas acerca da IU.

\section{MÉTODO}

Trata-se de uma pesquisa descritiva exploratória, transversal e com abordagem quantitativa. O estudo foi realizado na zona rural e urbana do município de Pilões, localizado no interior do Rio Grande do Norte. As participantes da pesquisa foram mulheres em faixa etária produtiva (20 a 59 anos). Foi escolhida essa faixa etária por essas mulheres estarem inseridas no processo produtivo/trabalho e por ser uma época de muito convívio social.

Considerando que a população da pesquisa é de 1.200 mulheres (IBGE, $2010)^{9}$ e aplicando-se o erro amostral de $5 \%$, a amostra foi composta por 300 mulheres. Tendo como critérios de inclusão: a) mulheres residentes na zona urbana ou rural do município de Pilões, b) ter entre 20 e 59 anos de idade e critérios de exclusão: a) ser portadora de algum comprometimento cognitivo ou mental. b) não estar presente em domicílio no momento da pesquisa.

A coleta de dados foi realizada mediante questionário, o qual foi auto aplicado com exceção dos casos em que as mulheres não sabiam ler e/ou escrever. As participantes assinaram o Termo de Consentimento Livre e Esclarecido- TCLE e foi entregue o questionário. No momento do recolhimento do questionário foi entregue um folheto educativo contendo informações referentes aos sinais e sintomas, causas e tratamento da IU.

A análise foi realizada por meio estatística descritiva, os resultados foram tabulados utilizando o programa IBM SPSS Statistics $20 ®$ e foi feito um teste de associação de QI-quadrado em Pearson entre as mulheres que se classificaram com IU e as que não se classificaram, mas não foi observada associação significativa entre as variáveis.

O estudo foi submetido a apreciação pelo Comitê de Ética e pesquisa da Universidade do Estado do Rio Grande do Norte (CEP UERN), sendo aprovado em 15 de março de 
2016, número do parecer: 1.451 .863 e

CAAE: 52945016.1.0000.5294.

\section{RESULTADOS}

A frequência de IU feminina na fase produtiva encontrada, no município de Pilões/RN, foi de $8 \%$, a queixa foi referida por 24 das 300 mulheres participantes.

Se extrapolarmos os dados para o total da população desta faixa etária, as 1.200 mulheres de 20 a 59 anos da zona urbana e rural, conforme censo (IBGE, $2010)^{9}$ pode-se estimar assim que 96 mulheres podem estar acometidas por IU nesse município.

A média de idade das mulheres com IU foi de $38,8 \pm 11,7$ anos. A média da renda familiar dessas mulheres foi de $1.321 \pm 1.435$ reais e a média de peso das mesmas foi de $64,9 \pm 10,6$ quilos.

Quanto ao perfil socioeconômico e demográfico, observou-se que 45,8 \% têm ensino médio completo e/ou incompleto, $54,1 \%$ são solteiras, $62,5 \%$ não trabalham, $83,3 \%$ residem na zona urbana, $62,5 \%$ residem em domicilio próprio, $66,6 \%$ tem seis ou mais cômodos e que $54,2 \%$ tem de duas a três pessoas que moram em seu domicílio.
A média de vezes que as mulheres com IU urinam durante o dia foi de $6,5 \pm 1,6$ e durante a noite foi de $1,8 \pm 0,98$ vezes.

Entre as mulheres com IU auto referida 20,8\% disseram que faziam algo que hoje não fazem, destas 54,2\% deixaram de ir a festas, por vergonha da perda de urina; $41,6 \%$ tem perda de urina uma vez e/ou mais de uma vez na semana e $66,7 \%$ tem perda de urina em forma de jato (Tabela 1). Quando questionadas sobre o que é incontinência urinária $100 \%$ das mulheres disseram que não sabiam o que era.

As situações nas quais as mulheres com IU auto referida tem perda de urina são pequeno esforço $26 \%$, médio esforço $62 \%$ e grande esforço $12 \%$.

Quando questionadas sobre seus hábitos diários tivemos as seguintes respostas: $91,7 \%$ das participantes costumam sentar na calçada, 79,2\% costumam frequentar a igreja, 95,8\% vão ir ao supermercado, 52,2\% não vão a festas, $58,3 \%$ saem de sair de casa para passear, $95,8 \%$ vão na casa de amigos e/ou familiares e $20,8 \%$ faziam alguma coisa que hoje em dia não fazem.

Tabela 1: Sinais e sintomas de mulheres com IU de 20 a 59 anos, residentes em Pilões/RN, 2017.

\begin{tabular}{lccc}
\hline \multicolumn{1}{c}{ Variável } & $\mathbf{N}$ & $\%$ \\
Várias vezes ao dia & \multicolumn{1}{c}{ Freque da perda de urina } & & \\
Mais de uma vez por semana & 1 & 4,2 \\
\hline
\end{tabular}




\begin{tabular}{|c|c|c|}
\hline Uma vez na semana & 5 & 20,8 \\
\hline Dificilmente & 13 & 54,2 \\
\hline Total & 24 & 100 \\
\hline \multicolumn{3}{|c|}{ Quantidade da perda de urina } \\
\hline Molha só a calcinha & 15 & 62,5 \\
\hline Molha a calcinha e a roupa & 5 & 20,8 \\
\hline Molha a roupa e escorre na perna & 4 & 16,7 \\
\hline Total & 24 & 100 \\
\hline \multicolumn{3}{|c|}{ Características da perda } \\
\hline Jato & 16 & 66,7 \\
\hline Gotejamento & 7 & 29,2 \\
\hline De forma continua & 1 & 4,2 \\
\hline Total & 24 & 100 \\
\hline \multicolumn{3}{|c|}{ Utiliza proteção para conter urina } \\
\hline Não & 18 & 75,0 \\
\hline Permanentemente & 4 & 16,7 \\
\hline Ocasionalmente & 2 & 8,3 \\
\hline Total & 24 & 100 \\
\hline \multicolumn{3}{|c|}{ Tipos de proteção para conter urina } \\
\hline Protetor de calcinha & 3 & 12,5 \\
\hline Absorvente & 2 & 8,3 \\
\hline Não usa proteção & 19 & 79,1 \\
\hline Total & 24 & 100 \\
\hline \multicolumn{3}{|c|}{ Frequência de troca de protetor durante o dia } \\
\hline Uma & 1 & 4,1 \\
\hline Duas a três & 3 & 12,5 \\
\hline Não usa proteção & 20 & 83,3 \\
\hline Total & 24 & 100 \\
\hline \multicolumn{3}{|c|}{ Frequência da troca de protetores durante a noite } \\
\hline Uma & 3 & 12,5 \\
\hline Não usa proteção & 21 & 87,5 \\
\hline Total & 24 & 100 \\
\hline \multicolumn{3}{|c|}{ Vida sexualmente ativa } \\
\hline $\operatorname{Sim}$ & 14 & 58,3 \\
\hline Não & 9 & 37,5 \\
\hline Não respondeu & 1 & 4,1 \\
\hline Total & 24 & 100 \\
\hline \multicolumn{3}{|c|}{ Vontade de urinar durante a relação sexual } \\
\hline Sim & 2 & 8,3 \\
\hline Não & 12 & 50 \\
\hline Não respondeu & 10 & 41,6 \\
\hline Total & 24 & 100 \\
\hline \multicolumn{3}{|c|}{ Evita ter relações sexuais devido à perda de urina } \\
\hline $\operatorname{Sim}$ & 2 & 8,3 \\
\hline Não & 12 & 50 \\
\hline Não respondeu & 10 & 41,6 \\
\hline Total & 24 & 100 \\
\hline \multicolumn{3}{|c|}{ Sua vida sexual ficou prejudicada devido à perda de urina } \\
\hline Sim & 1 & 4,1 \\
\hline Não & 13 & 54,1 \\
\hline Não respondeu & 10 & 41,6 \\
\hline Total & 24 & 100 \\
\hline
\end{tabular}

Fonte: própria

Dentre os hábitos investigados, $\quad 79,2 \%$ não praticam atividade física, $83,3 \%$ verificou-se que $87,5 \%$ não são fumantes, não fazem dieta, $91,7 \%$ consomem café e 83,3\% não fazem uso de bebida alcoólica, $50 \%$ consomem frutas cítricas (Tabela 2). 
Tabela 2: Hábitos de consumo alimentar e práticas de proteção à saúde das mulheres com IU auto referida de 20 a 59 anos, residentes em Pilões/RN, 2017.

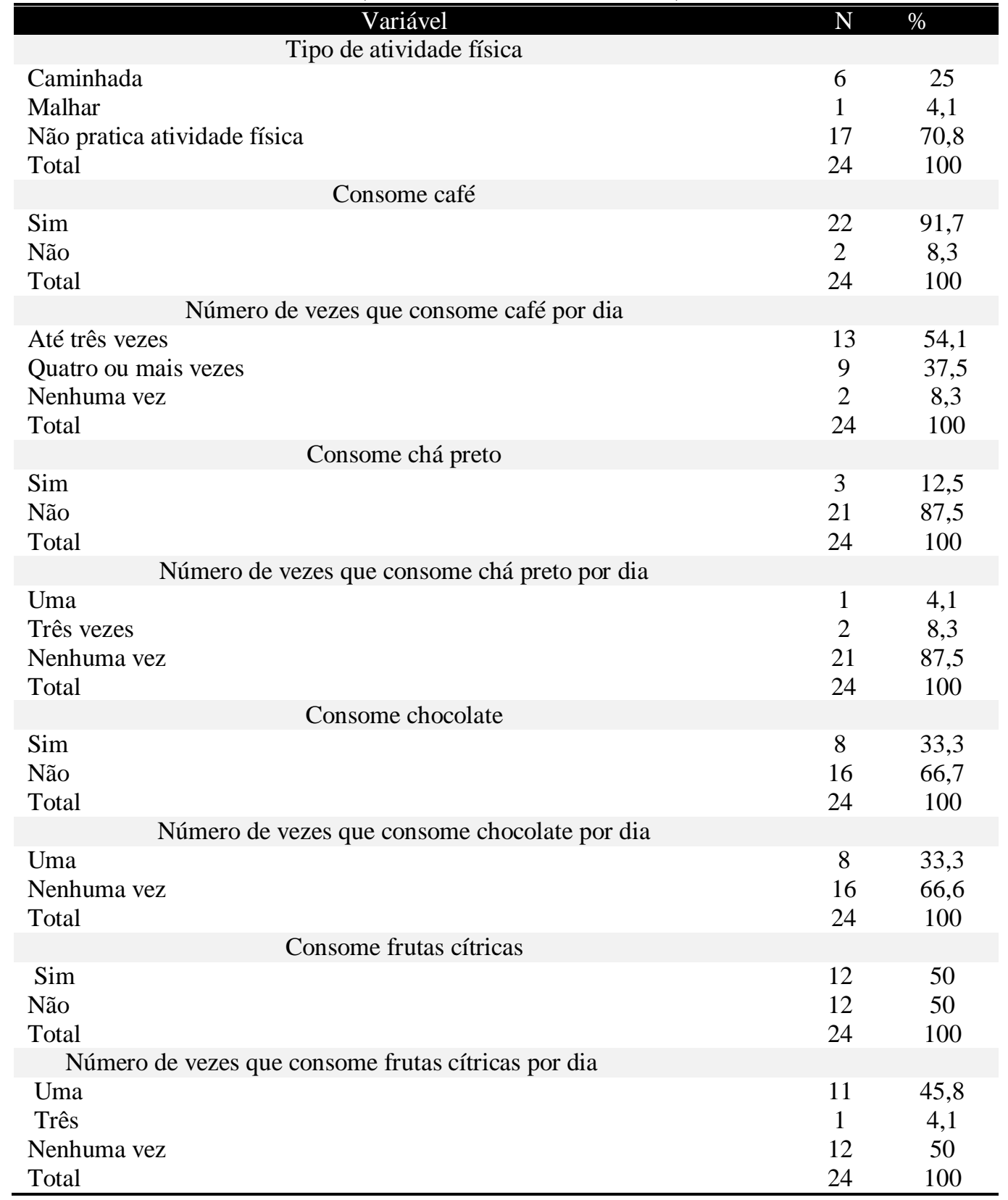

Fonte: própria

A média de peso, ao nascer, dos filhos das participantes da pesquisa foi de $3,5 \pm$ quilos 638 gramas. Quanto aos tipos de partos e quantidade de filhos das mulheres com IU, destaca-se que 70,8\% têm filhos; $58,3 \%$ são multíparas, $50 \%$ tiveram partos normais e que destas $37,5 \%$ tiveram dois ou mais partos normais (Tabela 3). 
Tabela 3: Tipos de partos e quantidade de filhos de mulheres com IU de 20 a 59 anos, residentes na zona urbana, residentes em Pilões/RN, 2017.

\begin{tabular}{|c|c|c|c|}
\hline \multirow{2}{*}{\multicolumn{4}{|c|}{$\begin{array}{l}\text { Variável } \\
\text { Número de filhos }\end{array}$}} \\
\hline & & & \\
\hline Primípara & & 3 & 12,5 \\
\hline Multípara & & 14 & 58,3 \\
\hline Nulipara & & 7 & 29,1 \\
\hline Total & & 24 & 100 \\
\hline \multicolumn{4}{|c|}{ Tipo de parto } \\
\hline Normal & & 12 & 50 \\
\hline Casaria & & 5 & 20,8 \\
\hline Sem partos & & 7 & 29,1 \\
\hline \multirow[t]{2}{*}{ Total } & & 24 & 100 \\
\hline & Total de partos normais & & \\
\hline Um & & 5 & 20,8 \\
\hline Dois ou mais & & 9 & 37,5 \\
\hline Sem partos & & 10 & 41,6 \\
\hline \multirow[t]{2}{*}{ Total } & & 24 & 100 \\
\hline & Total de partos Cesáreos & & \\
\hline Um & & 5 & 20,8 \\
\hline Dois & & 2 & 8,3 \\
\hline Sem partos & & 17 & 70,8 \\
\hline Total & & 24 & 100 \\
\hline
\end{tabular}

Fonte: própria

\section{DISCUSSÃO}

Aproximadamente 13 milhões de adultos nos Estados Unidos da América já vivenciaram algum episódio de IU, sendo que 11 milhões (85\%) são mulheres. ${ }^{10}$ Semelhante aos nossos achados Martins, Santos, Dorcínio, Alves, Roza e Luz (2017) 10 trazem que de acordo com os estudos realizados a experiência com episódios de perda urinária é uma condição que não prevalece somente em mulheres idosas, mas também mulheres jovens e na meia-idade.

Achado que aproximasse ao nosso estudo, o qual traz a estimativa de que 8$58 \%$ da população feminina adulta geral apresenta sintomas de incontinência, sendo que a hiperatividade vesical atinge aproximadamente $40 \%$ das mulheres que procuram atendimento médico. No Brasil, quase $10 \%$ das mulheres que visitam o ginecologista têm como queixa principal a perda de urina. $^{2}$

Fonseca, Galdino, Guimarães e Alves $(2010)^{4}$ encontrou entre as mulheres uma média de frequência urinária diurna de 3 vezes e noturna de 1,5 vezes, dados muito próximos aos nossos também. E apesar da IU levar a um aumento tanto da frequência urinária diurna quanto da noturna. Acreditase que a explicação para esse achado é o fato da maioria das pacientes do estudo apresentarem IU de esforço e não IU de urgência, sendo que esta última está mais relacionada com um aumento significativo da frequência urinária. 
O número de mulheres que referiram perda de urina em forma de jato representam um achado importante, uma vez que demonstra a gravidade da IU que essas mulheres são acometidas. Deste modo, nos alertou acerca do quão difícil é ter IU e enfrenta tal patologia. Similar aos nossos achados Figueiredo, Lara, Cruz, Quintão e Monteiro (2008) ${ }^{11}$ demonstram que $46 \%$ das participantes apresentaram perda em jato, $28 \%$ em gotas e $14 \%$ perda completa de urina, ou seja, perda de grande quantidade de urina associada à sensação de esvaziamento completo da bexiga.

Em relação aos sentimentos vivenciados pelas mulheres com IU alguns estudos, ${ }^{12,10,13,14,15}$ trazem que os danos psicológicos que a IU traz para as mulheres acometidas causam aflição e incapacidades, fato este que têm levado a significativa morbidade devido a sentimentos negativos de vergonha, medo de sofrer rejeição, constrangimento, solidão, tristeza, depressão, culpa, humilhação, ansiedade, irritação, falta de concentração, sensação de voltar a infância e medo de exalar cheiro de urina.

Vale ressaltar que parte considerável das mulheres com IU evitam ter relações sexuais devido à perda de urina e referiu que a vida sexual ficou prejudicada devido à perda de urina. Podemos perceber que a IU causa uma pior qualidade de vida para as mulheres acometidas pela patologia. Semelhante aos nossos achados Rett, Wardini, Santana, Mendonça, Alves e Saleme $(2016)^{15}$ dizem que a IU causam restrições no convívio social e sexual. Faria, Moraes, Monnerat, Verediano, Hawerroth e Fonseca $(2015)^{16}$ dizem que a IU tem um grande impacto negativo na qualidade de vida das mulheres que são acometidas pela doença afetando vários aspectos da vida dessas mulheres entre eles a higiene, a vida sexual. Traz também custos relacionados a absorventes e fraldas geriátricas, tendo impacto sobre o orçamento familiar.

Salienta-se que a maioria das mulheres que referiram IU, relatou não ter havido mudanças em seu convívio social, mas sim em seus hábitos, como o uso de protetores de calcinha por algumas, para conter a perda de urina. Este fato corrobora com o estudo de Rett, Wardini, Santana, Mendonça, Alves e Saleme (2016) ${ }^{15}$ que citam que a vivência com a IU leva a mulher realizar os mais diversos mecanismos de modificações comportamentais para se adaptar as inconveniências da perda urinária como: uso frequente de perfumes de odor forte; uso de absorventes ou protetores para controle da perda urinária; utilização de roupas escuras; diminuição do consumo hídrico; suspensão por conta própria de fármacos que estimulem a 
eliminação urinária; procura imediata pelo banheiro em locais públicos.

Destaca-se que nenhuma participante sabia o que era IU, deste modo, podemos observar a falta de conhecimento da população em geral a respeito das patologias que não estão na mídia e/ou não estão em epidemia. Em relação a este aspecto Leroy, Lúcio e Lopes (2016) ${ }^{7}$ traz que a maioria das mulheres com IU não tem ciência de que seus sintomas podem ser melhorados e curados com tratamento médico adequado. Outro aspecto relevante do estudo é o subdiagnóstico das mulheres com IU, que é decorrente do desconhecimento, das mesmas, a respeito da patologia, do tratamento. Nenhuma das participantes que referiram IU procuraram o médico para relatarem as perdas de urina. Em relação a este achado Fonseca et al. $(2005)^{17}$ traz que entre 30 e $50 \%$ das mulheres que sofrem de IU não relatam espontaneamente esse fato ao médico ou à enfermeira, e só procuram o serviço de saúde após o primeiro ano do início dos sintomas por acharem que a perda de urina é esperada com o evoluir da idade.

Em relação a este fato, alguns estudos 18,19,7,20 trazem que do total de mulheres incontinentes, a grande maioria consideram um problema muito pequeno, poucas consultam o médico ou qualquer outro profissional de saúde sobre esse problema.
Das mulheres que não comunicaram ao médico os motivos mais comuns são: vergonha e/ou falta de liberdade de falarem desse problema com o médico, considerar que é um acontecimento natural do envelhecimento e por esta razão não se ter o que fazer, pensar que passaria espontaneamente ou que não é suficientemente importante para consultar seu médico. Achado este que corroboram com os dados da nossa pesquisa.

Por conseguinte, foi observado um índice considerável de IU principalmente de esforço e a mesma traz impactos negativos para a qualidade de vida dessas mulheres. Para facilitar o diagnóstico e contribuir com um melhor acompanhamento, torna-se necessário que os profissionais de saúde abordem em suas consultas questionamentos referentes ao sistema gênito-urinário e a perda de urina, isso poderia ser posto em prática durante as consultas rotineiras, durante a consulta de crescimento e desenvolvimento da criança (C e D), exame Papanicolau, planejamento familiar. Para que isto aconteça é importante que os profissionais se capacitem a respeito da patologia.

\section{CONCLUSÃO}

Portanto, o presente estudo nos proporcionou conhecer a frequência da incontinência urinária em mulheres em 
idade produtiva na cidade de Pilões/RN, que foi de $8 \%$ número bastante relevante quando se leva em consideração a população total do município.

O mesmo nos levou a concluir que infelizmente todas as mulheres que não tem conhecimento sobre o que é a IU. Vale ressaltar que, apesar da incontinência urinária ser uma patologia que acomete mulheres nas mais variadas faixas etárias ainda é desconhecida.

\section{REFERÊNCIAS}

1. Santini ACM, Santos ES, Vianna LS, Bernardes JM, Dias A. Prevalência e fatores associados à ocorrência de incontinência urinária durante a gravidez. Rev Bras Saúde

Mater Infantil [Internet]. dez 2019 [citado em 04 fev 2020]; 19(4):967-74. Disponível em:

http://www.scielo.br/scielo.php?script=sci _arttext\&pid=S1519-

$38292019000400967 \& \operatorname{lng}=e n$. Epub 13 jan 2020. http://dx.doi.org/10.1590/180693042019000400013.

2. Kessler M, Facchini LA, Soares MU, Nunes BP, França SM, Thumé

E. Prevalência de incontinência urinária em idosos e relação com indicadores de saúde física e mental. Rev

Bras Geriatr Gerontol. [Internet]. ago 2018 [citado em 05 fev 20]; 21(4):397-

407. Disponível em: https://www.scielo.br/pdf/rbgg/v21n4/pt_1 809-9823-rbgg-21-04-00397.pdf.

http://dx.doi.org/10.1590/198122562018021.180015.

3. Berlezi EM, Fiorin AAM, Bilibio PVF, Kirchner RM, Oliveira KR. Estudo da incontinência urinária em mulheres climatéricas usuárias e não usuárias de medicação anti-hipertensiva. Rev Bras
Geriatr Gerontol. [Internet]. 2011 [citado em 05 fev 20]; 14(3):415-23. Disponível em:

https://www.scielo.br/pdf/rbgg/v14n3/v14n 3a02.pdf

4. Fonseca DC, Galdino DAA, Guimarães LHCT, Alves DAG. Avaliação da qualidade do sono e sonolência excessiva diurna em mulheres idosas com incontinência urinária. Rev Neurocienc. [Internet]. 2010 [citado em 05 fev 2020]; 18(3):294-99. Disponível em: http://www.revistaneurociencias.com.br/ed icoes/2010/RN1803/492\%20original.pdf

5. Sociedade Brasileira de Urologia. O que é a cirurgia de sling na incontinência urinária feminina? [Internet]. Rio de Janeiro: SBU; [201-?] [citado em 31 nov 2015]. Disponível em: https://portaldaurologia.org.br/publico/faq/ o-que-e-a-cirurgia-de-sling-naincontinencia-urinaria-feminina/

6. Higa R, Lopes MHBM. Fatores associados com a incontinência urinária na mulher. Rev Bras Enferm. [Internet]. 2005 [citado em 05 fev 2020]; 58(4):422-

28. Disponível em: https://www.scielo.br/pdf/reben/v58n4/a08 v58n4.pdf

7. Leroy LS, Lúcio A, Lopes MHBM. Risk factors for postpartum urinary incontinence. Rev Esc Enferm USP. [Internet]. 2016 [citado em 05 fev 2020]; 50(2):200-7. Disponível em: https://www.scielo.br/pdf/reeusp/v50n2/00 80-6234-reeusp-50-02-0200.pdf. doi: http://dx.doi.org/10.1590/ S0080623420160000200004

8. Ferreira M, Santos PC. Impacto dos programas de treino na qualidade de vida da mulher com incontinência urinária de esforço. Rev Port Saúde Pública [Internet]. 2012 [citado em 05 fev 2020]; 30(1):3-10. Disponível em: http://www.scielo.mec.pt/pdf/rpsp/v30n1/v 30n1a02.pdf

9. Instituto Brasileiro de Geografia e Estatística. Estimativas da população residente nos municípios brasileiros 
[Internet]. Rio de Janeiro: IBGE; [201-] [citado em 22 nov 2012]. Disponível em: https://www.ibge.gov.br/estatisticas/sociai s/populacao/9103-estimativas-depopulacao $\cdot h t m l$ edicao $=17283 \& \mathrm{t}=$ downlo ads

10. Martins LA, Santos KM, Dorcínio MBA, Alves JO, Roza T, Luz SCT. A perda de urina é influenciada pela modalidade esportiva ou pela carga de treino? uma revisão sistemática. Rev Bras Med Esporte [Internet]. fev 2017 [citado em 10 mar 2020]; 23(1):73-7. Disponível em: http://www.scielo.br/scielo.php?script=sci _arttext\&pid=S151786922017000100073\&lng=en

11. Figueiredo EM, Lara JO, Cruz MC, Quintão DMG, Monteiro MVC. Perfil sociodemográfico e clínico de usuárias de serviço de fisioterapia uroginecológica da rede pública. Braz. j. phys. ther. [Internet]. 2008 [citado em 05 fev 2020]; 12(2):13642. Disponível em:

https://www.scielo.br/pdf/rbfis/v12n2/a10v $12 \mathrm{n} 2 . \mathrm{pdf}$

12. Higa R, Rivorêdo CRSF, Campos LK, Lopes MHM, Turato ER. Vivências de mulheres Brasileiras com incontinência urinária. Texto \& Contexto Enferm, [Internet]. out/dez 2010 [citado em $05 \mathrm{fev}$ 2020]; 19(4):627-35. Disponível em: https://www.scielo.br/pdf/tce/v19n4/04.p df

13. Zago AC, Fambrini MAS, Silva EPG, Vitta A, Conti MHS, Marini G. Prevalence and knowledge of urinary incontinence and possibilities of treatment among lowincome working women. Fisioter Mov. [Internet]. 2017 [citado em 11 mar 2020]; 30(Suppl 1):151-9. Disponível em:

http://www.scielo.br/scielo.php?script=sci _arttext\&pid=S0103-

51502017000500151\&lng=en

14. Volkmer C, Monticelli M, Reibnitz KS, Brüggemann OM, Sperandio FF.

Incontinência urinária feminina: revisão sistemática de estudos qualitativos. Ciênc Saúde Colet. [Internet]. 2012 [citado em 05 fev 2020]; 17(10):2703-15. Disponível em: https://www.scielo.br/pdf/csc/v17n10/19.p df

15. Rett MT, Wardini ÉB, Santana JM, Mendonça ACR, Alves AT, Saleme CS. Female urinary incontinence: quality of life comparison on reproductive age and postmenopausal period. Fisioter Mov. [Internet]. mar 2016 [citado em 11 mar 2020]; 29(1):71-8. Disponível em: http://www.scielo.br/scielo.php?script=sci _arttext\&pid=S0103$51502016000100071 \& \operatorname{lng}=\mathrm{en}$

16. Faria CA, Moraes JR, Monnerat BR, Verediano KA, Hawerroth PA, Fonseca SC. Impacto do tipo de incontinência urinária sobre a qualidade de vida de usuárias do Sistema Único de Saúde no Sudeste do Brasil. Rev Bras Ginecol Obstet. [Internet]. 2015 [citado em 11 mar 2020]; 37(8):374-80. Disponível em: https://www.scielo.br/pdf/rbgo/v37n8/01 00-7203-rbgo-37-08-00374.pdf

17. Fonseca ESM, Camargo ALM, Castro RA, Sartori MGF, Fonseca MCM, Rodrigues de Lima G, Girão MJBC. Validação do questionário de qualidade de vida (King's Health Questionnaire) em mulheres brasileiras com incontinência urinária. Rev Bras Ginecol Obstet. [Internet]. 2005 [citado em 11 mar 2020]; 27(5):235-42. Disponível em: https://www.scielo.br/pdf/rbgo/v27n5/25 638.pdf

18. Oliveira E, Zuliani LMM, Ishicava J, Silva SV, Albuquerque SSR, Souza AMB, Barbosa CP. Avaliação dos fatores relacionados à ocorrência da incontinência urinária feminina. Rev Assoc Méd Bras. [Internet]. 2010 [citado em 05 fev 2020]; 56(6):688-90.

Disponível em: https://www.scielo.br/pdf/ramb/v56n6/v5 6n6a19.pdf

19. Carneiro JA, Ramos GCF, Barbosa ATF, Medeiros SM, Lima CA, Costa FM, et al. Prevalência e fatores associados à incontinência urinária em idosos não institucionalizados. Cad Saúde Colet. 
[Internet]. jul 2017 [citado em 11

mar 2020]; 25(3):268-77. Disponível em:

http://www.scielo.br/scielo.php?script=sci

_arttext\&pid=S1414-

462X2017000300268\&lng=en

20. Tomasi AVR, Santos SMA, Honório GJS, Locks MOH. Incontinência urinária em idosas: práticas assistenciais e proposta de cuidado âmbito da atenção primária de saúde. Texto \& Contexto Enferm.

[Internet]. 2017 [citado em 11 mar 2020]; 26(2):e6800015. Disponível em:

http://www.scielo.br/scielo.php?script=sci

_arttext\&pid=S0104-

07072017000200316\&lng=en

RECEBIDO: 14/01/2019

APROVADO: $17 / 07 / 2020$

PUBLICADO: $12 / 2020$ 